\title{
Cross-cultural adaptation and validation of the RhinAsthma Patient Perspective (RAPP) in the Polish population
}

\author{
Maciej Kupczyk ${ }^{1}$, Ilaria Baiardini², Giorgia Molinengo ${ }^{3}$, Barbara Loera ${ }^{3}$, Dorota Szydłowska ${ }^{1}$, Piotr Kuna ${ }^{1}$, \\ Giorgio Walter Canonica ${ }^{2,4}$, Fulvio Braido ${ }^{5}$
}

\author{
${ }^{1}$ Department of Internal Medicine, Asthma and Allergy, Medical University of Lodz, Lodz, Poland \\ ${ }^{2}$ Department of Biomedical Sciences, Humanitas University, Milan, Italy \\ ${ }^{3}$ Department of Psychology, University of Turin, Turin, Italy \\ ${ }^{4}$ Personalized Medicine, Asthma and Allergy Clinic, Humanitas Research Hospital, Milan, Italy \\ IIRCCS Ospedale Policlinico San Martino, Università di Genova, Dipartimento di Medicina Interna (DiMI), Genova, Italy
}

Adv Dermatol Allergol 2020; XXXVII (1): 97-102

DOI: https://doi.org/10.5114/ada.2020.93387

\begin{abstract}
Introduction: The RhinAsthma Patient Perspective (RAPP) was developed in Italian to assess the Health Related Quality of Life (HRQoL) impairment in patients with asthma and allergic rhinitis (AR) in daily practice.

Aim: To cross-culturally validate the Polish version.

Material and methods: The Polish version was administered to patients suffering from asthma and rhinitis in a prospective observational study. Polish RAPP, along with SF-12, ACT, and a Symptomatologic VAS was filled in twice, with a 4-week interval between visits. At visit 2, a Global Rating Scale (GRS) was completed to assess any change in health status. Internal consistency, validity, reliability, discriminant ability and responsiveness to change as well as Minimal Important Difference were determined.

Results: The factor and confirmatory analysis revealed a unidimensional structure of RAPP. Internal consistency was satisfactory with Cronbach's $\alpha$ (visit $1=0.85$, visit $2=0.89$ ). High reliability $(I C C=0.89$ and a CCC $=0.94)$ was found. Validity analyses showed good correlations of the Polish RAPP with Physical and Mental Component Scores of SF-12. In addition, RAPP adequately discriminated patients on the basis of the asthma control level and rhinitis severity ( $p<0.03$ for all the analyses), and demonstrated to be sensitive to change. MID value was 1 point. Conclusions: The study confirmed the reliability and validity of the Polish version of RAPP demonstrating that it is a useful tool in the assessment of HRQoL in patients with asthma and comorbid allergic rhinitis, in clinical practice.
\end{abstract}

Key words: allergic rhinitis, Health Related Quality of Life, questionnaire, validation study.

\section{Introduction}

Over the last few decades, there has been a great expansion in the use of Patient Reported Outcomes (PROs) as an indicator of subjective experience in asthma and allergic rhinitis (AR), mainly for research purposes [1, 2].

PROs are defined by the U.S. Food and Drug Administration as "any report of the status of a patient's health condition that comes directly from the patient, without interpretation of the patient's response by a clinician or anyone else" [3]. Collecting PROs enhances the range of patient outcomes that can be evaluated beyond the traditional clinical and biological measures.
In the field of respiratory allergy, among PROs, great attention has been dedicated to Health Related Quality of Life (HRQoL), a broad and multidimensional construct that refers to the impact of an illness and its treatment on a patient, as perceived by the patients themselves [4]. This is evident by the large number of specific questionnaires being validated $[5,6]$, and increasing assessment of this outcome when comparing groups of individuals in clinical trials and population studies.

The available data indicate that AR and asthma markedly affect the physical, emotional and social dimensions of patients' experience $[7,8]$. This is supported by evi-

Address for correspondence: Giorgia Molinengo PhD, Department of Psychology, University of Turin, Turin, Italy, e-mail: giorgia.molinengo@unito.it

Received: 18.04.2019, accepted: 29.05.2019. 
dence that successful treatment of respiratory allergy improves the HRQOL [9-13].

The implementation of PROs in clinical practice has recently been encouraged because of their potential to empower patients to self-manage their care [14], to support communication and partnership between physicians and patients, to affect care and outcomes, to improve patient satisfaction [15].

Unlike in other chronic conditions [16-18], the use of PROs in routine medical care with patients with respiratory allergy remains limited. This depends mainly on the lack of questionnaires with the necessary psychometric properties $[19,20]$ and practical characteristics [21] to support individual application.

The RhinAsthma Patient Perspective (RAPP) [22] has been developed and validated for the individual assessment of HRQoL keeping in mind the close relationship between asthma and comorbid AR [23-25]. It takes into account both upper and respiratory tracts allowing the capture of patients' experience in clinical practice.

The RAPP satisfies all the psychometric requirements that are requested for use in a routine setting [26]. Moreover, it is a highly practicable and user-friendly tool. The simple scoring system and the availability of a cut-off point with high sensitivity and specificity in discriminating the achievement of an optimal HRQoL, ensure that immediate feedback is available. This allows clinicians to integrate HRQoL results into their daily practice.

The RAPP has been validated in Italy [22] and is now available also in Portuguese [27]. To date, it has been the only available tool that can be used in clinical settings to monitor HRQoL of patients with respiratory allergy.

\section{Aim}

The aim of this study was therefore to cross-culturally adapt the Polish version of RAPP on the basis of proposed guidelines [28] and to test its psychometric properties. This project formed part of a larger international study whose purpose was to test the psychometric properties of the RAPP in five languages (Spanish, French, Portuguese, Polish and English) and to make comparison about HRQoL in the involved countries.

\section{Material and methods}

\section{Cross-cultural adaptation of the original RAPP into Polish}

The original Italian version of the RAPP was translated into Polish by two independent Polish native speakers fully competent in both languages. They were asked that the translation of each item should be semantic rather than literal, to reach conceptual and linguistic equivalence. Backward translation was performed by two native Italian speakers with fluent Polish and blind to the original Italian version of the RAPP. A consensus meeting of all researchers including translators was held to assess the semantic and conceptual aspects and to resolve any discrepancies, ambiguities and problems.

\section{Validation of the Polish version of the RAPP}

Patients who visited the allergy outpatient clinic of the Barlicki University Hospital, Medical University of Lodz (Poland), between March 2017 and October 2018 were invited to participate in the study.

The Ethics Committee of the University of Genoa approved the study protocol (approval no. P.R. 333REG2016), that was also ratified by a local ethics committee. The protocol complies with the general principles of Good Clinical Practice and the Declaration of Helsinki as amended in Edinburgh in 2000. Participation was voluntary and anonymous, and informed consent was obtained from all patients before study entry.

The inclusion criteria were as follows: age $>18$ years, literate native Polish speakers and having been diagnosed with asthma and AR according to GINA [29] and ARIA [30] guidelines.

Participants were excluded in case of the presence of other respiratory or ear-nose-throat disorders.

Each patient included in the study was examined at two different visits.

During the first visit the physician collected socio-demographic and anthropometric data, information about disease patterns, spirometry values, smoking habits, and current treatment. Patients were asked to fill in the Polish version of the RAPP along with three other PRO measures:

- Short Form Survey (SF-12) [31], a validated tool for the assessment of health status. It is composed of 12 items providing two domain scores: a physical component score (PCS) and a mental component score (MCS), with sum score ranges from 0 (the worst possible health) to 100 (the best possible health).

- Asthma Control Test (ACT), a validated questionnaire, widely used to assess the level of asthma control within the 4 weeks preceding the evaluation [32]. The tool consists of five questions that evaluate limitations in daily activities, amount of dyspnoea, the presence of nocturnal symptoms, the use of rescue medication and perceived asthma control. Patients assign a score from 1 (poorest control) to 5 (total control). The resulting ACT score is interpreted as follows: fully controlled asthma (score $\geq 25$ ), poorly to partially controlled asthma (score 20-24), or uncontrolled asthma (score < 20) [10].

- Symptomatologic Visual Analog Scale (VAS) [33]: a 100-mm long horizontal line in which patients indicate the global discomfort due to their AR during the previous week from 0 (not at all bothersome) to 100 (intolerably bothersome) (100 mm). The Joint Task Force on Practice Parameters proposed this simple measure to assess the AR symptom severity [22]. 
On the second visit, 4 weeks later, the Polish RAPP, the SF-12, ACT and Vas were collected. In order to assess any change in health status, patients were also asked to fill in a Global Rating Scale.

Between the visits all patients received treatment according to the current GINA and ARIA guideline recommendations.

The psychometric properties of the Polish version of RAPP were evaluated by means of consistency, reliability and validity statistical analysis.

The internal consistency of RAPP items was tested using Cronbach's $\alpha$ coefficient, considering values greater than 0.70 acceptable [34], whereas higher scores are recommended for use in an individual patient [35]. Moreover, a sub-sample of patients with a stable health status (GRS $=0$ ) was selected to perform inter-class coefficient (ICC) and Lin's concordance correlation coefficient (CCC) in order to study scale reliability. For these coefficients a rule of thumb of 0.70 for group comparisons, and of 0.90 for comparisons within individuals is recommended [36].

Exploratory and confirmatory factor analysis [37, 38] models were estimated to test scale dimensionality. To assess the confirmative model fit, three indexes were considered: the root-mean-square error of approximation (RMSEA), the standardized root mean squared residual (SRMR) and the comparative fit index (CFI).

Values from 0.4 to 0.8 of the Pearson's correlation between the RAPP and SF-12 scores was intended as a test of convergent validity, while the group comparison of patients (ANOVA) derived from ACT, GINA and ARIA classification of severity served to test scale discriminant validity.

Scale responsiveness was evaluated by analysing the correlation between changes in RAPP scores and changes in GRS, VAS and ACT by means of Pearson's correlation coefficients.

Finally, the minimal important difference, MID [39], was determined using the receiver operating characteristics (ROC) curve, with the adoption of the entire cohort for one dichotomization point (i.e., 'no change' vs. 'any improvement or deterioration').

In addition, the possible effect of the education and smoking habits on patients' answers was controlled using ANOVA, age effect was explored through correlation analysis, and to verify that RAPP properly represented patients' quality of life levels the frequency distribution of scores was inspected.

\section{Statistical analysis}

IBM SPSS Statistics (version 24, Armonk, NY) and Mplus 7.0 (Muthén \& Muthén, Los Angeles, CA) were used.

\section{Results}

The study involved 127 patients, slight female majority $(53.5 \%)$ with a mean age of 34.8 years (range: 18-66 years). The majority of patients had an academic degree (48\%) or high school diploma (39.4\%), while the remaining participants had a secondary school (9.4\%) and primary school (3.2\%) diploma. In terms of occupation, $74.8 \%$ were employees, $18.2 \%$ students, $3.9 \%$ unemployed and $3.1 \%$ retired. The prevalence of persistent asthma in the sample was $62.2 \%$. ACT scores at the first visit indicated that $12.5 \%$ were totally controlled, $57.5 \%$ were well controlled, and the remaining 30\% were uncontrolled. The mean value of $A R$ and asthma quality of life was 16.6 at first visit and 16.1 at second visit.

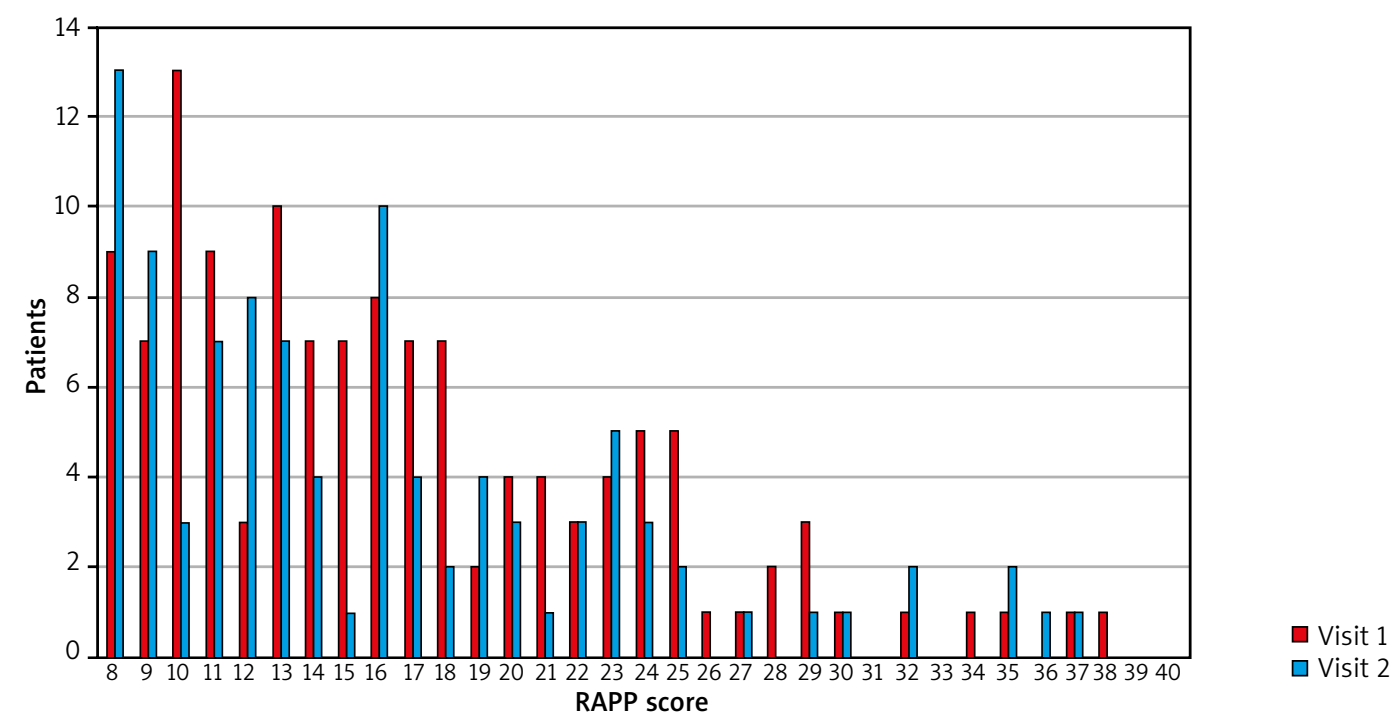

Figure 1. RAPP scores distributions at Visit 1 and Vist 2 
Table 1. RAPP discriminant validity

\begin{tabular}{|c|c|c|}
\hline \multirow[t]{2}{*}{ Variable } & \multicolumn{2}{|c|}{ RAPP score } \\
\hline & Visit 1 & Visit 2 \\
\hline \multicolumn{3}{|l|}{ Asthma: } \\
\hline Mild & $16.7(5.9)$ & $16.9(6.1)$ \\
\hline Moderate & $16.7(5.6)$ & $16.6(7.7)$ \\
\hline Severe & $16.5(7.8)$ & $15.4(8.0)$ \\
\hline$P$-value & NS & NS \\
\hline \multicolumn{3}{|l|}{ ACT: } \\
\hline Totally controlled & $11.9(4.1)$ & $13.5(5.4)$ \\
\hline Well controlled & $15.1(6.0)$ & $14.5(6.5)$ \\
\hline Uncontrolled & $21.5(7.1)$ & $20.8(8.2)$ \\
\hline$P$-value & $<0.001$ & $<0.001$ \\
\hline \multicolumn{3}{|l|}{ Rhinitis: } \\
\hline Intermittent & $14.2(5.7)$ & $14.8(6.8)$ \\
\hline Persistent & $17.9(7.2)$ & $16.7(7.2)$ \\
\hline$P$-value & $<0.001$ & 0.03 \\
\hline \multicolumn{3}{|l|}{ Rhinitis severity: } \\
\hline Moderate & $13.3(4.2)$ & $13.5(4.8)$ \\
\hline Severe & $18.8(7.5)$ & $18.1(8.3)$ \\
\hline$P$-value & $<0.001$ & 0.02 \\
\hline
\end{tabular}

The RAPP score distribution at the first and second visits is presented in Figure 1.

Cronbach's $\alpha$ values equal to 0.85 at the first visit and 0.89 at the second one, indicate an appreciable internal consistency. Reliability, assessed in 43 patients reporting stable health status (GRS $=0$ ), provides an ICC of 0.89 and a CCC value equal to 0.94.

The RAPP scale revealed a unidimensional structure that absorbed $43.6 \%$ of the total observed variance, and 1 residual greater than $|0.10|$ at the first visit. Data from the second visit remained stable, with $51 \%$ of the total variance explained, and 4 residuals greater than $|0.10|$. The same structure was obtained from confirmatory factor analysis: the model fit indexes were all satisfactory, both at first (RMSEA 0.08, SRMR 0.04 , CFI 0.94) and second visits (RMSEA 0.05, SRMR 0.04, CFI 0.98).

Convergent validity was achieved: correlations between RAPP scores and the Physical Component Score of SF-12 were significant at both first $(r=-0.49$, $p \leq 0.001)$ and second visit $(r=-0.28, p<0.001)$. Significant correlations were also found considering RAPP and the Mental Component Score of SF-12 (Visit 1:
Table 2. The MID of RAPP obtained with the ROC analysis with different cut-off values

\begin{tabular}{lcc}
\hline Cut-off $\geq$ & Sensitivity (\%) & Specificity (\%) \\
\hline 5 & 0.754 & 0.529 \\
\hline 4 & 0.755 & 0.411 \\
\hline 3 & 0.765 & 0.313 \\
\hline 2 & 0.876 & 0.247 \\
\hline $1^{*}$ & 0.893 & 0.189 \\
\hline${ }^{*}$ Cut-off point chosen. & \\
& \\
$r=-0.28, p<0.02 ;$ Visit 2: $r=-0.27, p<0.01)$. RAPP
\end{tabular}
results, presented in Table 1 , showed that the tool was able to discriminate between patients on the basis of the asthma control level and rhinitis severity $(p<0.03$ for all the analyses). Moreover, RAPP was significantly associated with VAS $(r=0.47, p<0.001)$ and ACT $(r=-0.46, p<0.001)$ in the sub-sample of 84 patients reporting an improvement or deterioration in health status.

A 1-point difference or change in RAPP (MID) maximizes sensitivity, specificity, and the number of individuals correctly classified (Table 2).

RAPP scores showed no significant differences between smokers, former smokers, and non-smokers (ANOVA Fisher's test: Visit 1: $p=0.25$; Visit 2: $p=0.58$ ), nor regarding age (Spearman's correlation: Visit 1: $r=-0.04$; Visit 2, $r=0.06$ ), nor level of education (ANOVA Fisher's test. Visit 1: $p=0.26$; Visit 2: $p=0.17$ ).

\section{Discussion}

RAPP is the first tool for individual asthma and rhinitis HRQoL assessment in daily practice. In this study, RAPP was cross-culturally adapted from the original Italian version $[22,40]$ to Polish with two forward and backward translation and its psychometric properties was assessed in 149 patients with asthma and rhinitis. Our findings are in line with previous validation procedures in Italian and Portuguese populations. The Polish RAPP proved the unidimensional structure of the original Italian questionnaire, as previously confirmed in the Portuguese version. In terms of internal consistency, it has a satisfactory performance, with Cronbach's $\alpha$ values that approach the recommended threshold for questionnaires to be used in clinical practice [35]. Test-retest reliability in patients reporting a stable health status was good (ICC $=0.89$ and a CCC $=0.94)$. Construct validity was confirmed by the correlation with the physical and mental component of health status. RAPP showed discriminative ability with respect to asthma control and AR severity and it is responsive to changes in health. The ROC analysis indicates that 1 point is the smallest change that patients perceive as an improvement or deterioration. Our MID value is half of the value found in Braido 
and Todo-Bom [22, 27]. This is in line with the fact that the MID value is not a fixed value but it may vary in relation with the features of the population considered. This difference could be explained considering the different population of patients enrolled in the study.

Patient's answers were not influenced by age, level of education or smoking habits, indicating that RAPP may be used in daily practice independently of patients' characteristics and behaviour.

\section{Conclusions}

The RAPP was successfully cross-culturally adapted and validated for use with Polish speaking patients. Its psychometric properties were similar to those of the original Italian version and Portuguese version. The Polish version of RAPP can be recommended as a robust tool questionnaire to be used in clinical practice to monitor HRQoL of patients with asthma and AR.

\section{Acknowledgments}

The authors thank ARMIA (Associazione Ricerca Malattie Immunologiche e Allergiche) for scientific support.

This work was supported by Menarini International Operations Luxemburg S.A. (M.I.O.L.).

\section{Conflict of interest}

The authors declare no conflict of interest.

\section{References}

1. Braido F, Bousquet PJ, Brzoza Z, et al. Specific recommendations for PROs and HRQOL assessment in allergic rhinitis and/or asthma: a GA(2)LEN taskforce position paper. Allergy 2010; 65: 959-68.

2. Cabana MD, Kunselman SJ, Nyenhuis SM, Wechsler ME. Researching asthma across the ages: insights from the National Heart, Lung, and Blood Institute's Asthma Network. J Allergy Clin Immunol 2014; 133: 27-33.

3. Patrick DL, Burke LB, Powers JH, et al. Patient reported outcomes to support medical product labeling claims: FDA perspective. Value Health 2007; 10 (Suppl. 2): S125-37.

4. Schipper H, Clinch J, Olweny CLM. Quality of life studies: definitions and conceptual issues. In: Quality of life and pharmacoeconomics in clinical trials. Spilker B (ed.). Lippincot-Raven Press, Philadelphia 1990; 11-23.

5. Apfelbacher CJ, Hankins M, Stenner P, Frew AJ, Smith HE. Measuring asthma-specific quality of life: structured review. Allergy 2011; 66: 439-57.

6. Worth A, Hammersley V, Knibb R, et al. Patient-reported outcome measures for asthma: a systematic review. NPJ Prim Care Respir Med 2014; 24: 14020.

7. Linneberg A, Dam Petersen K, Hahn-Pedersen J, et al. Burden of allergic respiratory disease: a systematic review. Clin Mol Allergy 2016; 14: 12
8. Meltzer EO. Allergic rhinitis: burden of illness, quality of life, comorbidities, and control. Immunol Allergy Clin North Am 2016; 36: 235-48.

9. Novakova SM, Staevska MT, Novakova PI, et al. Quality of life improvement after a three-year course of sublingual immunotherapy in patients with house dust mite and grass pollen induced allergic rhinitis: results from real-life. Health Qual Life Outcomes 2017; 15: 189.

10. Locks RB, Dos Santos K, da Silva J. Quality of life in patients with allergic rhinitis: a clinical trial comparing the use of bilastine versus loratadine. Clin Otolaryngol 2017; 42: 218-24.

11. Svedsater $\mathrm{H}$, Jones R, Bosanquet $\mathrm{N}$, et al. Patient-reported outcomes with initiation of fluticasone furoate/vilanterol versus continuing usual care in the Asthma Salford Lung Study. Respir Med 2018; 141: 198-206.

12. Scichilone N, Arrigo R, Paternò A, et al. The effect of intranasal corticosteroids on asthma control and quality of life in allergic rhinitis with mild asthma. J Asthma 2011; 48: 41-7.

13. Padilla Galo A, Labor M, Tiotiu A, et al. Impact of reslizumab on outcomes of severe asthmatic patients: current perspectives. Patient Relat Outcome Meas 2018; 9: 267-73.

14. Wintner LM, Giesinger JM, Kemmler G, et al. The benefits of using patient-reported outcomes in cancer treatment: an overview. Wien Klin Wochenschr 2012; 124: 293-303.

15. Chen J, Ou L, Hollis SJ. A systematic review of the impact of routine collection of patient reported outcome measures on patients, providers and health organisations in an oncologic setting. BMC Health Serv Res 2013; 13: 2119.

16. Biber J, Ose D, Reese J, et al. Patient reported outcomes experiences with implementation in a University Health Care setting. J Patient Rep Outcomes 2018; 2: 34.

17. Rotenstein LS, Agarwal A, O'Neil K, et al. Implementing patient-reported outcome surveys as part of routine care: lessons from an academic radiation oncology department. J Am Med Inform Assoc 2017; 24: 964-8.

18. Rotenstein LS, Huckman RS, Wagle NW. Making patients and doctors happier - the potential of patient-reported outcomes. N Engl J Med 2017; 377: 1309-12.

19. International Society for Quality of Life Research. User's Guide to Implementing Patient-Reported Outcomes Assessment in Clinical Practice. Available from: http://www.isoqol. org/UserFiles/2015UsersGuide-Version2.pdf. Version: January 2015. Accessed February 2019.

20. Frost MH, Reeve BB, Liepa AM, et al.; Mayo/FDA PatientReported Outcomes Consensus Meeting Group. What is sufficient evidence for the reliability and validity of patientreported outcome measures? Value Health 2007; 10 Suppl 2: S94-105.

21. Kroenke K, Monahan PO, Kean J. Pragmatic characteristics of patient-reported outcome measures are important for use in clinical practice. J Clin Epidemiol 2015; 68: 1085-92.

22. Braido F, Baiardini I, Stagi E, et al. RhinAsthma patient perspective: a short daily asthma and rhinitis QoL assessment. Allergy 2012; 67: 1443-50.

23. Bousquet J, Khaltaev N, Cruz AA, et al. Allergic Rhinitis and its Impact on Asthma (ARIA) 2008 update (in collaboration with the World Health Organization, GA(2)LEN and AllerGen). Allergy 2008; 63 Suppl 86: 8-160.

24. Halpern MT, Schmier JK, Richner R, et al. Allergic rhinitis: a potential cause of increased asthma medication use, costs, and morbidity. J Asthma 2004; 41: 117-26.

25. Shaaban R, Zureik M, Soussan D, et al. Rhinitis and onset of asthma: a longitudinal population-based study. Lancet Lond Engl 2008; 372: 1049-57. 
26. International Society for Quality of Life Research. User's Guide to Implementing Patient-Reported Outcomes Assessment in Clinical Practice. Available from: http://www.isoqol. org/UserFiles/2015UsersGuide-Version2.pdf. Version: January 2015. Accessed February 6, 2019.

27. Todo-Bom A, Braido F, Molinengo G, et al. The Portuguese version of Rhinitis and Asthma Patient's Perspective (RAPP): Validation and assessment. Pulmonology 2019 Feb 5. https://doi.org/10.1016/j.pulmoe.2018.10.009.

28. Wild D, Grove A, Martin M, et al. Principles of Good Practice for the Translation and Cultural Adaptation Process for Patient-Reported Outcomes (PRO) Measures: report of the ISPOR Task Force for Translation and Cultural Adaptation. Value Health 2005; 8: 94-104.

29. Global Initiative for Asthma (GINA) [homepage] [Accessed February 1, 2019]. Available from: http://ginasthma.org/.

30. Brozek JL, Bousquet J, Agache I, et al. Allergic Rhinitis and its Impact on Asthma (ARIA) Guidelines - 2016 Revision. J Allergy Clin Immunol 2017; 140: 950-8.

31. Gandek B, Ware JE, Aaronson NK, et al. Cross-validation of item selection and scoring for the SF-12 Health Survey in nine countries: results from the IQOLA Project. International Quality of Life Assessment. J Clin Epidemiol 1998; 51: 1171-8.

32. Nathan RA, Sorkness CA, Kosinski M, et al. Development of the asthma control test: a survey for assessing asthma control. J Allergy Clin Immunol 2004; 113: 59-65.

33. Bousquet PJ, Combescure C, Neukirch F, et al. Visual analog scales can assess the severity of rhinitis graded according to ARIA guidelines. Allergy 2007; 62: 367-72.

34. Cronbach LJ. Coefficient alpha and the internal structure of tests. Psychometrika 1951; 16: 297-334.

35. Scientific Advisory Committee of the Medical Outcomes Trust. Assessing health status and quality-of-life instruments: attributes and review criteria. Qual Life Res 2002; 11: 193-205.

36. Lin LI. A concordance correlation coefficient to evaluate reproducibility. Biometrics 1989; 45: 255-68.

37. Streiner DL. Health Measurement Scales: a Practical Guide to Their Development and Use. $3^{\text {rd }}$ edn. Oxford University Press, Oxford 2003.

38. Brown MW, Cudeck R. Alternative ways of assessing model fit. In: Testing Structural Equation Models. Bollen KA, Long JS (eds.). Newbury Park, Calif: Sage 1993; 136-62.

39. Turner D, Schunemann HJ, Griffith LE, et al. Using the entire cohort in the receiver operating characteristic analysis maximizes precision of the minimal important difference. J Clin Epidemiol 2009; 62: 374-9.

40. Molinengo G, Baiardini I, Braido F, Loera B. RhinAsthma patient perspective: a rasch validation study. J Asthma 2018; 55: 119-23. 